\title{
Structure character of timber frame system building in Tang and Song dynasty of China
}

\author{
Rui Gao, a , Lingqiang Yang ${ }^{1, b}$, Yan Wang ${ }^{1, c}$ \\ ${ }^{1}$ School of Civil engineer and architecture, University of Jinan, Jinan, Shandong province, China \\ acea_Gaor@ujn.edu.cn, cea_yanglq @ujn.edu.cn ${ }^{\text {b }}$,eo_wangy @ujn.edu.cn ${ }^{c}$
}

Keywords: palace building; timber frame system; China

Abstract. Chinese traditional palace is an important component of traditional architecture. Gold box bucket bottom slot hall-type building is a kind of the highest rank traditional architecture in China. The main structure consist of frontal column layer, tile layer and roof layer, in which, the connection between stigma and cap block, stigma and column base are yaw floating shelves. Under the horizontal inertia force, post partial pendulum and the weight of roof make it reset.

\section{Introduction}

Chinese traditional palace which penetrates and bandages in Chinese people's all aspects of life, is broad and profound. As an indispensable part of people's life, traditional buildings influenced by the traditional culture deeply. The palace building is an important component of traditional architecture. It is Chinese traditional architectural development stage in Tang dynasty and song dynasty, so far, Buddha temple east hall, only music temple Goddess of Mercy Pavilion, and Wooden Pagoda in Ying county, etc. are preserved, which are important physicals during the period of Tang dynasty and Liao dynasty ${ }^{[1]}$. These buildings are " bucket bottom slot hall-type ", which is the highest level of traditional building in wooden frame system in China. It's good anti-seismic performance represents construction technology achievement and level during this period. "Construction code of timber frame" was produced during Song dynasty, and then the building technology was to be further developed and improved. In order to explore the mystery of the excellent anti-seismic performance, which have pass through several earthquake during more than one thousand years, it is necessary to study the method of the construction inherent relations between these architectures and traditional architectures. So in the paper, the build mode of "golden box bucket bottom palace" without vice- order type frame was taken as example to study ${ }^{[2]}$.

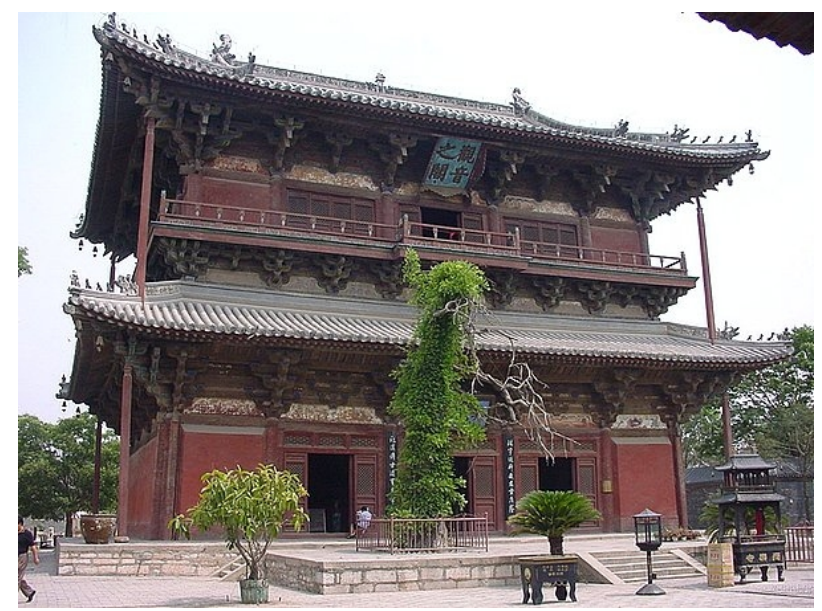

Fig. 1 Dule temple

\section{Structural Characteristics of The Frame System}

Under the tile layer and roof layer, plane column grid of gold box bucket bottom palace type construction is back glyph(Fig. 2), two laps column head both inside and outside are connected by architraves as a whole which forms column frontal layer(Fig. 3). 


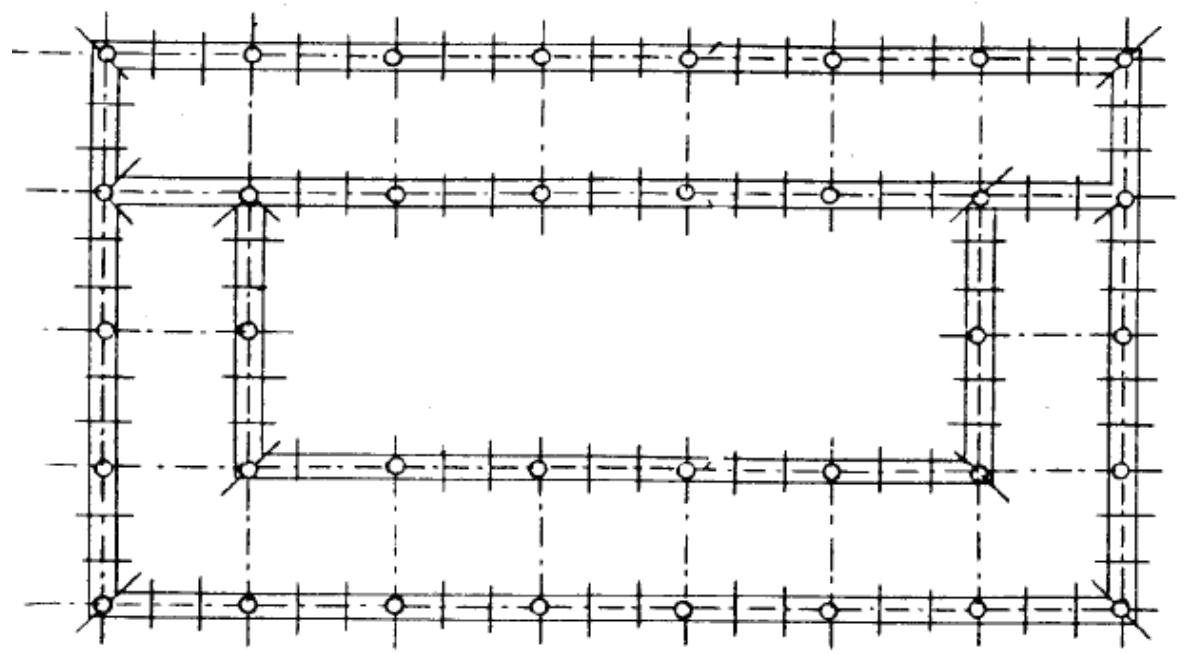

Fig. 2 the branch tank of gold box bucket bottom tank

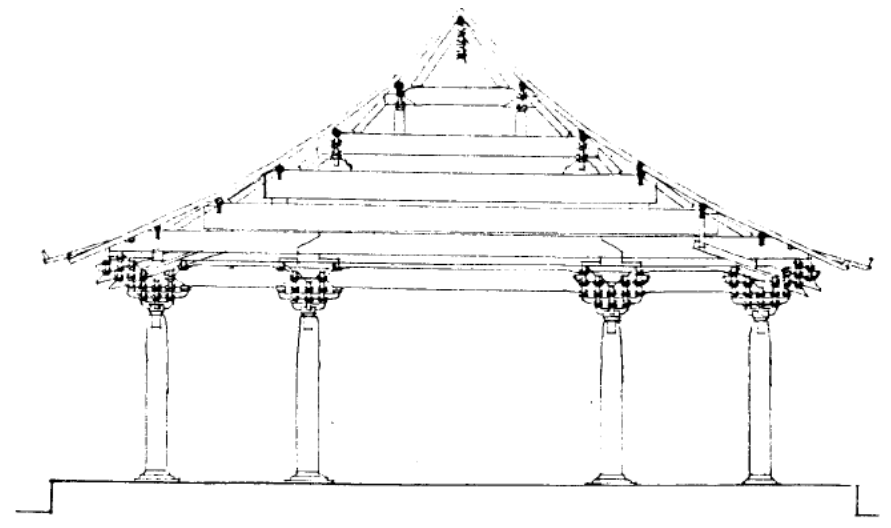

Fig. 3 The single eave hall-architecture stratified chart

\section{Roof layer}

Roof layer is consist of frame system and roof maintenance layer.Roof maintenance layer including roofing tile layer and basic layer which is composed of bamboo fence,reed foil,clay, and lime. Owing to the frame folded,roof maintenance layer is smooth surface and thicker ( the thickness can be up to 30 $\mathrm{cm}$ ), so roof longitudinal stiffness is strong.Frame system is consist of level one beams, superpositions as well as tuofeng,king posts,fork hands and supporting feet.There are many chuan in the longitudinal direction, panjian components and roof whose stiffness is strong connect together as a whole.so the roof layer in the longitudinal direction can be considered to be rigid.In the transverse direction,the system of the beam deformation of the layers of beams itself in the axial direction can be neglected, the kind of what lateral stiffness is mainly depends on whether each layer beams shift with each other.We take the flat beam and four rafters beam in cloth analysis (Fig. 4).Look at flat beam firstly,fork hand beam constitute triangle,ridge chuan of the triangle sit on Shu column across for wood and a set of bracket set,fixed by fork hands on both sides, on the other side of the fork hand insert head of flat beam by mortise ,two fork hands are stuck the upper approximately a quarter by Ding Hua wiping chin arch,reduced free length of the fork hand. When a horizontal force pressure on the roof,force transfer to beam mount through the chuanzi and chuan from mechanical point of view,Since among ridge chuan, for wood, arch and Shu column, can be simplified to the flat pendulum floating shelf, the out-of-plane frame members deformation binding is small. The construction state can be simplified as $4 \mathrm{a}$, as a result,fork hand mainly bear horizontal force $(\mathrm{F})$, one side of the fork hand be pulled, another pressed.It is mortise that between fork hands and the head of flat beams, the pulled fork hands pulled out from birch easily,such as fork hand AC point out from C (Fig. 5),so the horizontal force F can only be withstand by the other side of the pressed fork hands $(\mathrm{AB})$.It will certainly produce an upward force 
component at this moment, as a result,fork hands do the arc motion upwards around the flat beam head point B,But this upward force component are balanced by main ridge which is heavy and part of the roof self- weight actually. Arc motion of the fork hands will not occur, Therefore, the triangle ABC can remain stable and the shape similar to the rigid body.Horizontal force $\mathrm{F}$ can only make the triangle translation ,but flat beam head is sitting on the cap block which sit above the hump, it can against flat beam level movement relying on friction and promote the supporting feet $\mathrm{BD}$ at the same time.It is the same situation that between supporting feet and the fork hands above, because of the weight of the roof can not do arc movement as well,So it limits the relative displacement between flat beam and the four rafter beam ,forming a stable triangle $\square \mathrm{ADE}$ whose bottom edge is four rafters beams, that is to say, roof layer in the transverse can also be regarded as rigid.

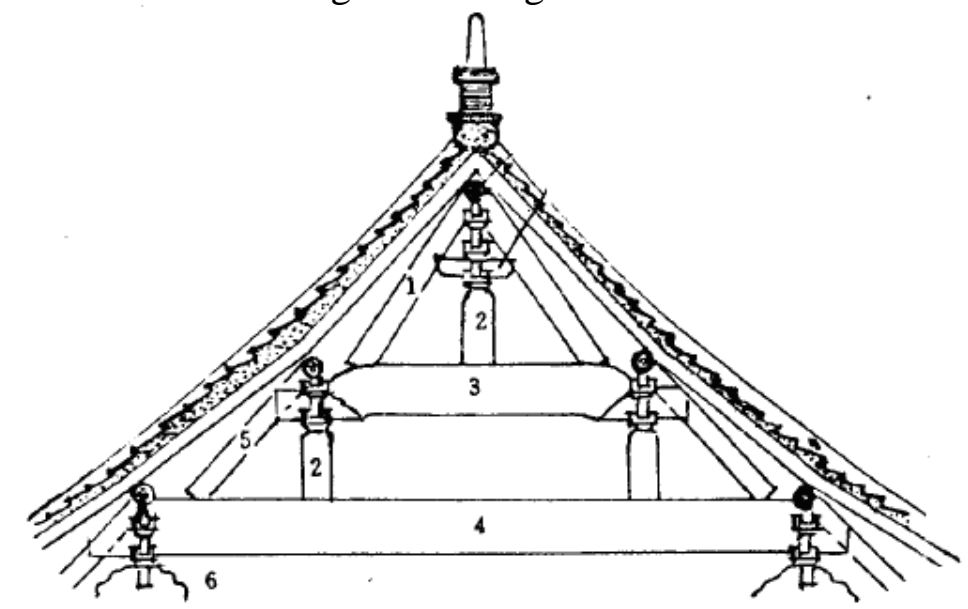

Fig. 4 simple roof truss structure

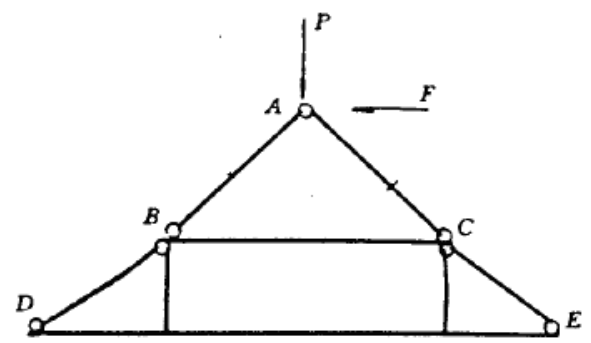

(a)

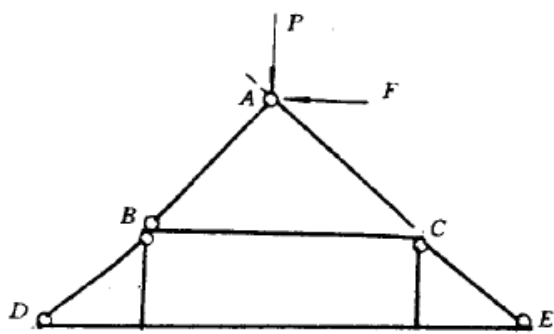

(b)

Fig. 5 Lateral stiffness of the roof beams

\section{Bracket set on columns layer}

Bracket set on columns layer consists of cap block,arm,lever and painted beam which interspersed in longitudinal and transverse ,bite each other,recede layer by layer,as shown in figure 5 .But architrave member engaging with each other only within on the same layer, their are all yaw floating resting between the upper and lower layers. There are liao eaves beam, pressure tank beam luohan beam, stigma beam etc in longitudinal of bracket set on columns layer,that can can make every duopu together into a whole, it is mingru beams in transverse (a part of bracket set on columns layer)that connected to the inside groove bracket set on columns layer and outer integrally,so that the bracket set on columns at stigma form an uniform grid-like bracket set on columns layer.Compared to the horizontal and vertical , lateral stiffness is weak(Because there are only mingru beam contacted in transverse between bracket set on columns but there are more connected members and bracket set on columns layers in vertical, it forms a vertical siding among seam on stigma, arch, painted beam, arch partitions, especially in the stigma seam.Its longitudinal stiffness coefficient stronger than transverse,according to the kinetic analysis.Therefore, measures were taken on the building 
construction,Adding partitioned ears within alternate cap block on flower arm jump head,to strengthen the capacity of its anti-lateral horizontal force.Even so,the calculation results still show that the longitudinal stiffness coefficient stronger than the transverse,It showed that bracket set on columns have a greater flexibility in the transverse.

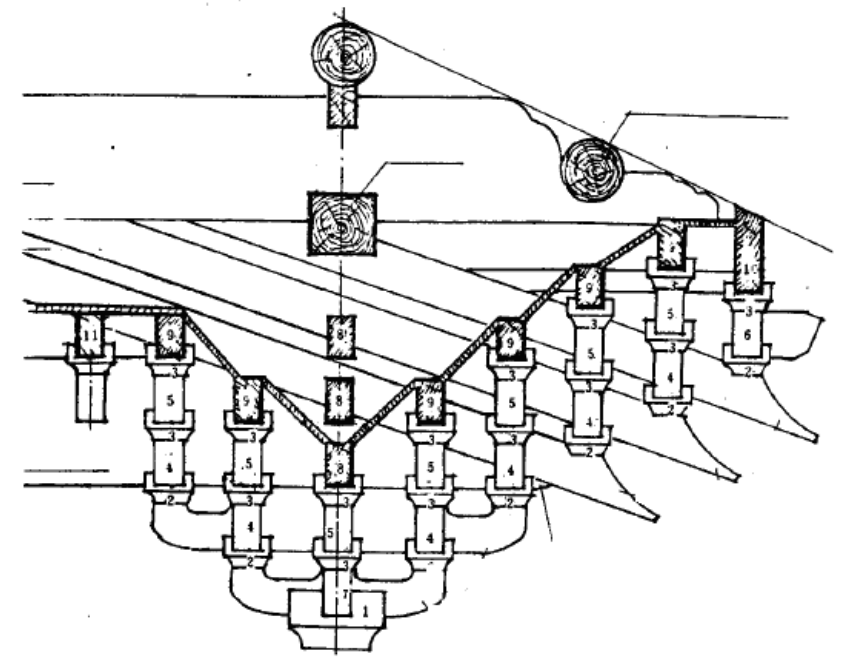

Fig. 6 Bracket set on columns structure of stigma

\section{Frontal column layer}

The frontal column layer of palace type building without vice order is composed of beam , architrave and ground beam(parts of that does not have ground beam). There are mene and Sill wall, etc, generally. (such as buddha temple hall), but in lateral,there is no support member between columns, to resist horizontal seismic forces relying on stigma and column foot with their respective contact surface friction, that is to say, the friction between the the stigmas column head and cap block, column feet and column base, so the stiffness coefficient is small. As can be seen by the above analysis, lateral framework is weak zone in the hall-type buildings, especially frontal column layer,it is also instability and failure under horizontal force,so reliable measures must be taken in constructions of these buildings.

\section{Conclusions}

The roof layer is a rigid, to ensure normal use of roofing maintenance layer;column amount layer is flexible and provides sufficient deformation capacity;bracket set on columns layer is the nexus transitive layer, in three components of the hall-frame system, three components unified with each other as a whole, reflecting the concept of considering structural system integrally.

\section{Acknowledgements}

This work was financially supported by the Shandong Natural Science Foundation (ZR2014EL038).

\section{References}

[1] Chinese Academy of Sciences Institute. History of Chinese ancient architecture technology. Science Press. 1990.

[2] Conservationtech Consulting . Learning from the past to protect the future: Armature Crosswalls. Engineering Structures 30 (2008) 2096-2100. 